\title{
INVESTIGATION INTO CAVITATION INDUCED NOISE WITHIN HYDRAULIC RELIEF VALVE
}

\author{
Ji Hong*, Fu Xin*, Yang Huayong*, Tetsuhiro Tsukiji** \\ *State Key Laboratory of Fluid Power Transmission and Control \\ Zhejiang University \\ Zheda Street, HangZhou, 310027,China \\ (E-mail: xfu@sfp.zju.edu.cn) \\ ** Department of Mechanical Engineering \\ Sophia University \\ 7-1, Kioi-cha, Chiyoda-ku, Tokyo, 102-8554, Japan
}

\begin{abstract}
Cavitation commonly occurred in hydraulic components is generally regarded as harmful phenomena in the hydraulic control systems, because the collapse of the cavitation bubbles could induce the vibration, noise and some times even corrupt severely the surface of the elements. Motivated by a desire to suppress cavitation induced noise in hydraulic control valve, the objective of this work is to study the inception of the cavitation and to evaluate the effects of internal structure of the valve on cavitation intensity as well as cavitation noise. In the presented paper, the computational fluid dynamics and flow visualization approaches are used to obtain static pressure distributions and cavitation images in the channel of the main stage of the relief valve. The predicted low-pressure regions and gas volume fraction of cavitation agree well with the observed cavitation areas. The noise level from the traditional and optimized internal structures of the relief valves are tested and compared.
\end{abstract}

\section{KEY WORDS}

Relief valve, Flow field simulation, Cavitation, Noise control

\section{INTRODUCTION}

Relief valve is one of the major noise sources in fluid power systems. A great attention has been paid to understand the mechanism of the flow noise and to develop the practical technique of noise control. Traditional approach of stability analyses as well as advanced approach of computational fluid dynamics is used to achieve optimum internal structure and a better noise performance of the hydraulic relief valves, as reported in the literature ${ }^{[1-4]}$.

It is generally accepted that the cavitation is of prime cause for inducing the noise. Petroleum-based oil contains $6 \sim 12 \%$ air in volume. When the pressure of oil is below the air separation pressure, the air nucleuses expand to form cavitation. In high pressure region downstream, the bubbles are collapsed causing pressure oscillation and noise.

In the presented paper, the computational fluid dynamics and flow visualization approaches are used to obtain static pressure distributions and cavitation images in the channel of the main stage of the relief valve. The predicted low-pressure regions and gas volume fraction agree well with the observed cavitation areas and pattern. The method of the flow field control is used to optimize the internal structure of the relief valve. The noise level from the traditional (Fig. 1 (a)) and optimized (Fig.1 (b)) internal structures of the relief valves are tested and compared. The result shows that the main element with circumferential undercut groove can suppress the cavitation and cavitation noise. The internal structure

\footnotetext{
* The project is supported by National Nature Science Foundation of China (599086)
} 
optimization is therefore proved an available strategy for the low noise design of hydraulic relief valve.

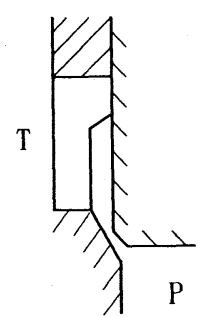

(a)

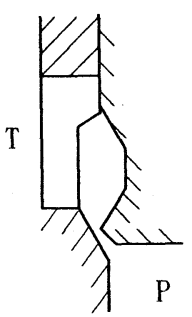

(b)
Fig. 1 Valve internal geometry

\section{FLUID FIELD COMPUTATION}

Internal geometry around the orifice of the relief valve is axi-symmetric and an axi-symmetric 2-D geometry model can model the components. The three dimensional entrance and exit region of the valve body is ignored as shown Fig.2. Line $\mathrm{OO}$ is axis, $\mathrm{OA}$ is inlet and $\mathrm{BB}$ is outlet. ACDB and BF is the wall of valve body, and OEF is the boundary of the plug. The computation domain is divided into three mesh regions. Region 2 uses the smallest mesh size since the pressure and velocity vary greatly in this area. Fig. 3 shows the local fine mesh near orifice.

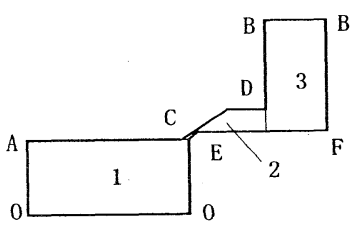

(a) Type A

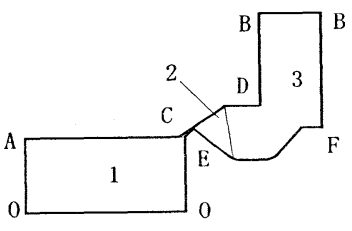

(b) Type B
Fig. 2 Computation domain

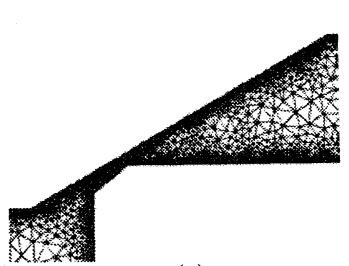

(a)

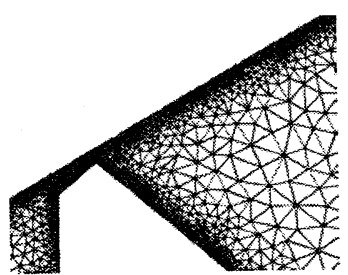

(b)
Fig .3 Local fine meshes

In the computation, the valve stroke is $0.5 \mathrm{~mm}$, the mean inlet velocity is $2.8 \mathrm{~m} / \mathrm{s}$ and the outlet pressure is set $0.1 \mathrm{Mpa}$. The oil density is $890 \mathrm{~kg} / \mathrm{m}^{3}$ and kinetic viscosityv $40 \times 10^{-6} \mathrm{~m}^{2} / \mathrm{s}$. The inlet velocity distribution is supposed parabolic. The inlet velocity $\mathrm{u}$ is known as:

$u=\frac{\Delta p}{4 \mu \cdot l}\left(\frac{d^{2}}{4}-r^{2}\right)$

where $d$ is diameter, $r$ is radius variable of pipe, $\triangle p$ is pressure drop between two end of pipe, $\mu$ is fluid dynamic viscosity and $l$ the pipe length.

Integrating Eq. (1) on the inlet section gives Eq. (2) as below

$q=\frac{\pi \cdot d^{4} \cdot \Delta p}{128 \mu \cdot l}$

Substituting Eq. (2) into Eq. (1) gives

$u=\frac{32 q}{\pi \cdot d^{4}}\left(\frac{d^{2}}{4}-r^{2}\right)$

Where $q$ is flow rate.

Eq. (3) determines the inlet velocity by the inlet flow rate.

In the flow field calculation, the commercial software FLUENT 5.5 was used. Adopted cavitation model in the software, some parameters are set as follow, vaporization pressure is $0.033 \mathrm{Mpa}$, bubble number density $1.8 \times 10^{7}$ $1 / \mathrm{m}^{3}$. The calculation results are given as Fig. 4 and Fig. 5.


(b)

Fig .4 Contours of absolute pressure

Fig. 4 shows the pressure distributions around the orifices of the valves. In Fig. 4 (a) low pressure areas lies just behind chamfer of plug, from -2.1 to 0.14 , because of flow detachment from wall caused by high velocity in orifice. After small distance from orifice downstream the pressure increase to 0.77 . In Fig. 4 (b) the pressure behind orifice enhance relatively. A large vortex is observed and the low pressure of $0.19 \mathrm{Mpa}$ occurs in the center of the 
vortex. As the intensity of the cavitation is related to the pressure, the increase of the lowest pressure in Fig.4 (b) could lead to a slighter cavitation. The resistances of the orifices are also different in the two structures at identical opening. At the same pressure drop between inlet and outlet, for instance $1.8 \mathrm{Mpa}$, in Fig.4 (b) the flow rate is about $52 \mathrm{~L} / \mathrm{min}$ and in Fig. 4 (a) the flow rate is about $67 \mathrm{~L} / \mathrm{min}$, which indicates that the large vortex results in high pressure loss and dissipates more flow kinetic energy. Therefore, the noise level of the structure (b) could be lower than that of structure (a).

Fig. 5 shows the contours of gas volume fraction in valve chamber, its patterns and locations are remarkable different. In Fig.5 (a) the gas locates mainly behind chamfer of plug, gas volume fraction decrease rapidly from 0.4 to 0.017 along flow, while in Fig.5 (b) high fraction of gas volume focus on the center of vortex.

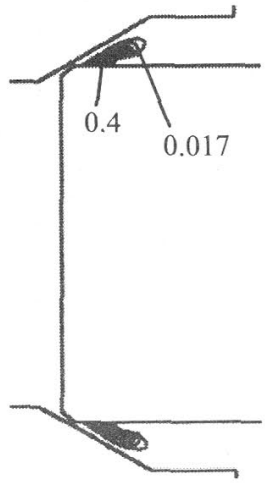

(a)

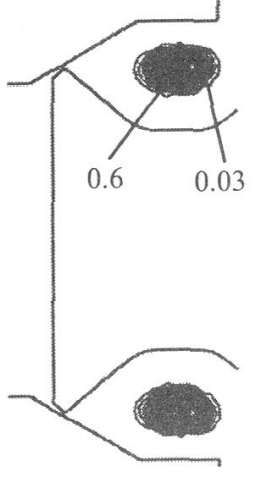

(b)
Fig .5 Contours of gas volume fraction

\section{EXPERIMENTAL STUDY AND RESULTS}

In order to validate the calculated results, the experiments are conducted by the means of cavitation visualization and noise measurement.

\subsection{Experimental Equipment}

Fig. 6 shows the diagram of experimental system. Hydraulic power unit is insulated with valve model and noise measurement apparatus to depress its noise influence. Tube 3 and hydraulic attenuator 4 are used to insulate the mechanical vibration and absorb pressure fluctuation in experimental system. The proportional relief valve 1 is used as a safety value in the system. Regulating the pump 2 can control the flow rate to valve model.

The valve bodies are made by the transparent material acrylic as shown in Fig. 7. The valve opening can be regulated to simulate the different operation condition. The operation pressure is under 5MP to avoid the damage of the acrylic valve body.

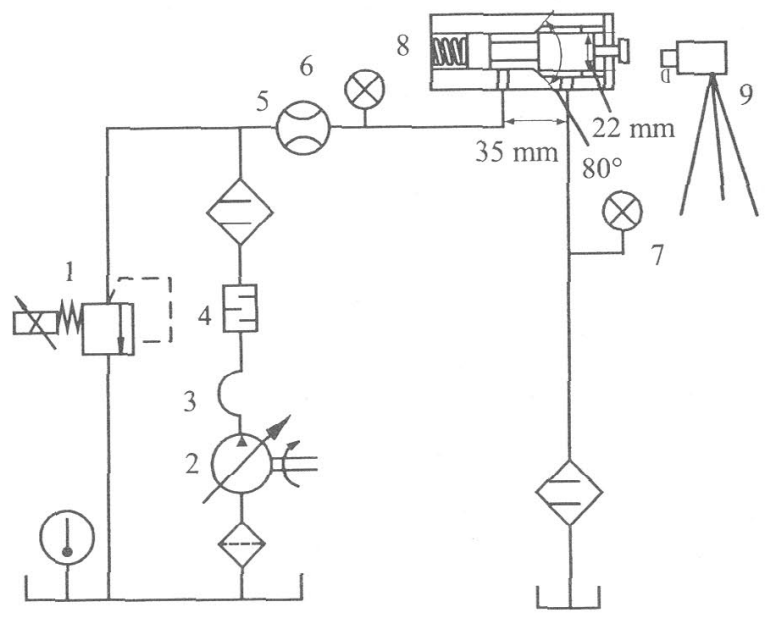

Fig .6 Sketch of experiment system

1-proportional relief valve 2-proportional variable pump 3-tube 4-hydraulic attenuator 5-flow meter 6, 7-pressure transducer 8-valve model 9-fine noise measure apparatus

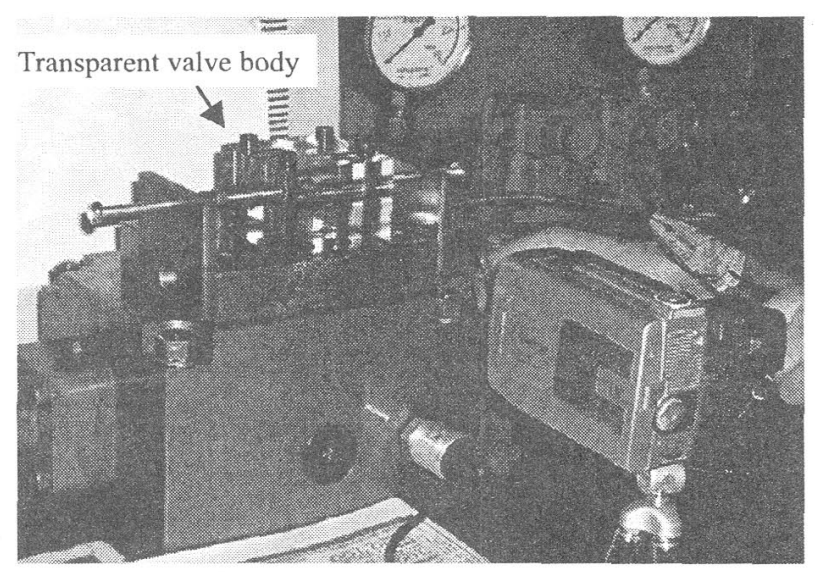

Fig .7 Experimental apparatus

\subsection{Results of Cavitation Visualization}

The cavitation phenomena in two types of different internal geometries and a series of different operation conditions are observed. Fig.8 gives two of typical cavitation images under the operation condition listed in table 1 .

From Fig. 8 it can be seen that the location and pattern of cavitation are different in the two internal structures. As shown in Fig.8 (a), cavitation occurs in relatively low inlet pressure. It is born in the exit of the orifice and aggregates in the downstream of the orifice. The bubbles 
are dense and small. For the structure with groove on valve plug, however, the cavitation does not occur dramatically at the exit of the orifice, as see in Fig. 8 (b). In this case, the cavitation exists in the center of the large chamber. Its bubbles are large and thin. In experiment, a large vortex is seen in the chamber because of the bubbles motion. It seems the location of cavitation is in the center of vortex where the pressure is the lowest.

Table 1 Experiment conditions of visualization

\begin{tabular}{c|c|c|c|c}
\hline No. & $\begin{array}{c}\text { Inlet press. } \\
{[\mathrm{MPa}]}\end{array}$ & $\begin{array}{c}\text { Outlet press. } \\
{[\mathrm{MPa}]}\end{array}$ & $\begin{array}{c}\text { Flow rate } \\
{[\mathrm{L} / \mathrm{min}]}\end{array}$ & $\begin{array}{c}\text { Noise } \\
{[\mathrm{dB}]}\end{array}$ \\
\hline $\mathrm{a}$ & 2.06 & 0.1 & 29.2 & 80 \\
$\mathrm{~b}$ & 1.79 & & 30.6 & 74 \\
\hline
\end{tabular}

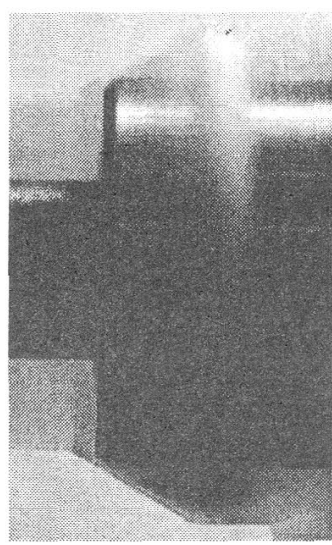

(a)

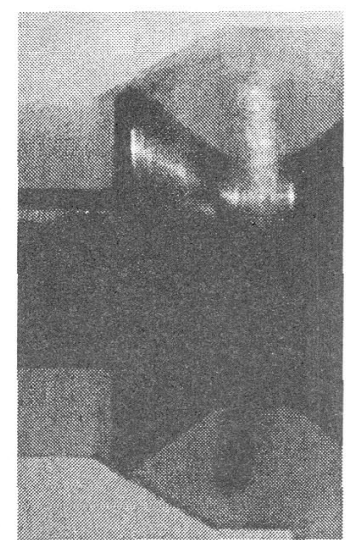

(b)
Fig 8 Cavitations images

Comparing the experiment result shown in Fig. 8 with the simulation results shown in Fig.4 and Fig.5, one can find that features of the cavitation relate to the form of the vortex. The cavitation always exists in the centre of the vortex. When the vortex is intense enough, the centrifugal force of the vortex will pull the small bubbles, which are far lighter than oil into the centre of the vortex forming large bubbles.

\subsection{Result of Noise Measurement}

The noise levels of two kinds of the geometry of valves are measured for different openings and flow rates. The background noise is about $63 \mathrm{~dB}$ (A weighted). The microphone for the measurements is set at a distance of $50 \mathrm{~mm}$ from valve body. The typical measurement results are shown in Fig.9.

In Fig.9, curve $q$ shows the relationship between flow rate and inlet pressure, curve $L_{\text {eq }}$ shows the relationship between noise level and inlet pressure, and the valve opening $h$ is about $0.2 \mathrm{~mm}$. Geometry type $\mathrm{A}$ is the valve model without undercut groove and geometry type B is the valve model with undercut groove. From Fig.9, it can be seen that the noise of the geometry type B is lower in average than that of geometry type $\mathrm{A}$. The results indicate that the undercut groove enhances the vortex and forces the bubbles of cavitation to be congregate into the center of the vortex. The congregation of the cavitation avoids the direct explosion and concussion of the air bubbles to the wall of the valve body and therefore the noise level of the geometry type B is reduced.

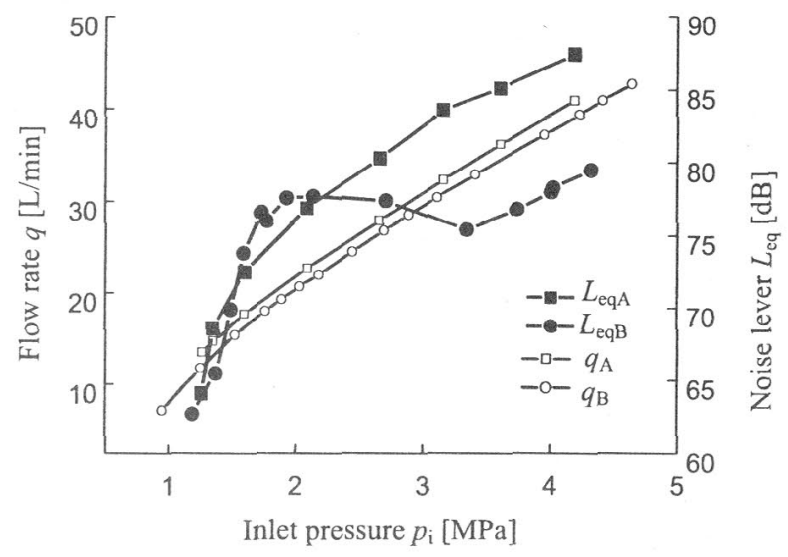

Fig .9 Experimental results of noise level

\section{CONCLUTIONS}

(1) The valve plug with circumferential undercut groove can change cavitation location and pattern, which reduce effectively noise level of the relief valve.

(2) The valve plug with circumferential undercut groove causes large and strong vortex in the flow channel, which can increase the flow resistance, suppress the inception of cavitation and dissipate flow kinetic energy, resulting in the reduction of the noise level.

\section{REFERENCES}

1.Hisanori Ueno, Noise measurement and numerical simulation of oil flow in pressure control valves, JSME International Journal, series b, vol.37, no.2, 1994.

2.H. Nguyen-schaefer, Study on the flow in a typical seat valve of mobile hydraulic, SAE Special Publications v 1229 Feb. 1997. p 11-22 970812.

3.Borghi M., Milani M., Paoluzzi, R, Transient flow force estimation on the pilot stage of a hydraulic valve, The Fluid Power and Systems Technology Division (Publication) FPST v 5 p 157-162, ASME, 1998.

4.Horinouchi, Katsutoshi, Numerical study of flow cavitation, SAE Transactions v 99 n Sect 61990 p 1133-1140, 1990. 